TRANSACTIONS OF THE

AMERICAN MATHEMATICAL SOCIETY

Volume 351, Number 11, Pages 4657-4673

S 0002-9947(99)02307-7

Article electronically published on July 20, 1999

\title{
QUASITRIANGULAR + SMALL COMPACT $=$ STRONGLY IRREDUCIBLE
}

\author{
YOU QING JI
}

\begin{abstract}
Let $T$ be a bounded linear operator acting on a separable infinite dimensional Hilbert space. Let $\epsilon$ be a positive number. In this article, we prove that the perturbation of $T$ by a compact operator $K$ with $\|K\|<\epsilon$ can be strongly irreducible if $T$ is a quasitriangular operator with the spectrum $\sigma(T)$ connected. The Main Theorem of this article nearly answers the question below posed by D. A. Herrero.

Suppose that $T$ is a bounded linear operator acting on a separable infinite dimensional Hilbert space with $\sigma(T)$ connected. Let $\epsilon>0$ be given. Is there a compact operator $K$ with $\|K\|<\epsilon$ such that $T+K$ is strongly irreducible?
\end{abstract}

\section{INTRODUCTION}

Let $\mathcal{H}_{1}, \mathcal{H}_{2}, \mathcal{H}$ be separable Hilbert spaces. Denote by $\mathcal{B}\left(\mathcal{H}_{1}, \mathcal{H}_{2}\right)$ the set of all bounded linear operators mapping $\mathcal{H}_{1}$ into $\mathcal{H}_{2}$. Denote by $\mathcal{K}\left(\mathcal{H}_{1}, \mathcal{H}_{2}\right)$ the subset of $\mathcal{B}\left(\mathcal{H}_{1}, \mathcal{H}_{2}\right)$ of all compact operators. We simply write $\mathcal{B}(H)$ and $\mathcal{K}(H)$ instead of $\mathcal{B}(H, H)$ and $\mathcal{K}(H, H)$ respectively. For $T \in \mathcal{B}\left(\mathcal{H}_{1}, \mathcal{H}_{2}\right)$, denote the kernel of $T$ and the range of $T$ by $\operatorname{Ker} T$ and $\operatorname{Ran} T$ respectively. If $\mathcal{H}_{0}$ is a subspace of $\mathcal{H}$ (closed), we shall write $\mathcal{H}_{0} \leq H$. Let $T \in \mathcal{B}(H)$; we shall denote by $\sigma(T)$, $\sigma_{p}(T), \sigma_{l}(T), \sigma_{r}(T), \sigma_{e}(T), \sigma_{l e}(T), \sigma_{l r e}(T)$ and $\sigma_{w}(T)$ the spectrum, the point spectrum, the left spectrum, the right spectrum, the essential spectrum, the left essential spectrum, the Wolf spectrum and the Weyl spectrum of $T$ respectively. Denote by $\sigma_{0}(T)$ the set of all isolated points of $\sigma(T) \backslash \sigma_{e}(T)$. For $\lambda \in \rho_{S-F}(T) \stackrel{\text { def }}{=}$ $\left.\mathcal{C} \backslash \sigma_{\text {lre }}(T)\right), \operatorname{ind}(T-\lambda)=\operatorname{dimKer}(T-\lambda)-\operatorname{dim} \operatorname{Ker}(T-\lambda)^{*}$ and $\min \operatorname{ind}(T-\lambda)=$ $\min \left\{\operatorname{dimKer}(T-\lambda), \operatorname{dim} \operatorname{Ker}(T-\lambda)^{*}\right\}$. For $-\infty \leq n \leq+\infty, \rho_{S-F}^{(n)}(T)=\{\lambda \in$ $\left.\rho_{S-F}(T): \operatorname{ind}(T-\lambda)=n\right\}$. $T$ is said to be quasitriangular if there is a sequence $\left\{P_{n}\right\}_{n \geq 1}$ of finite rank projections increasing to the unit operator $I$ with respect to the strong operator topology such that $\lim _{n \rightarrow \infty}\left\|\left(I-P_{n}\right) T P_{n}\right\|=0$. It is well-known that $T$ is quasitriangular if and only if $\operatorname{ind}(T-\lambda) \geq 0$ for all $\lambda \in \rho_{S-F}(T)$. T is said to be strongly irreducible if there are no nontrivial idempotents commuting with $T$. A Cowen-Douglas operator is an operator $T$ satisfying the following conditions:

(i) There is a nonempty connected open subset $\Omega$ of $\rho_{S-F}^{(n)}(T)$ for a natural number $n$.

(ii) $T-\lambda$ is surjective for each $\lambda \in \Omega$.

(iii) $\bigvee\{\operatorname{Ker}(T-\lambda): \lambda \in \Omega\}$ is equal to the acting space of $T$.

Received by the editors May 23, 1997.

1991 Mathematics Subject Classification. Primary 47A10, 47A55, 47A58.

Key words and phrases. Weyl spectrum, index, strongly irreducible, quasitriangular.

This work is supported by MCSEC. 
If the conditions above are satisfied, we shall write $T \in \mathcal{B}_{n}(\Omega)$. If $T \in \mathcal{B}_{n}(\Omega)$, then $\bigvee\left\{\operatorname{Ker}(T-\lambda)^{k}: k \geq 1\right\}$ is equal to the acting space of $T$ for each $\lambda$ in $\Omega$.

Let $\sigma$ be a compact subset of the complex field $\mathcal{C}$. A clopen $\sigma_{0}$ of $\sigma$ is a subset of $\sigma$ such that there are two disjoint open subsets $\Omega_{1}, \Omega_{2}$ of $\mathcal{C}$ such that $\Omega_{1} \supset \sigma_{0}$ and $\Omega_{2} \supset\left(\sigma \backslash \sigma_{0}\right)$.If $\sigma$ is a clopen of $\sigma(T)$, then there is an analytic Cauchy domain $\Omega$ such that $\sigma(T) \cap \Omega=\sigma$ and such that $\sigma(T) \cap \partial \Omega=\emptyset$, where $\partial \Omega$ is the boundary of $\Omega$. Thus $E(\sigma, T)=\frac{1}{2 \pi i} \int_{\partial \Omega}(\lambda-T)^{-1} d \lambda$ is an idempotent commuting with $T$. We call $E(\sigma, T)$ the Riesz idempotent of $T$ corresponding to $\sigma$. Write $\mathcal{H}(\sigma, T)=$ $\operatorname{Ran} E(\sigma, T)$. It follows from the classical Riesz decomposition theorem that $T$ is not strongly irreducible if $\sigma(T)$ is not connected. But the converse is not true. However, D. A. Herrero and C. L. Jiang obtained the approximate inverse of the Riesz decomposition theorem (see [3] or [6]):

Theorem HJ. The closure of the class of all strongly irreducible operators is the class of all those operators which have connected spectrum.

And then, D.A. Herrero posed the following question.

Question H. Let $T$ be an operator with $\sigma(T)$ connected. Given $\epsilon>0$, can we find a compact operator $K$ with $\|K\|<\epsilon$ such that $T+K$ is strongly irreducible?

C.L. Jiang, S.H. Sun and Z.Y. Wang (see [10]) proved that if $T$ is essentially normal and if $\sigma(T)$ is connected, then one can find a compact $K$ such that $T+K$ is strongly irreducible. (But $\|K\|$ may be bigger than $\epsilon$.) Y.Q. Ji, C.L. Jiang and Z.Y. Wang (see [8], [9]) proved that if $T$ is an essentially normal quasitriangular operator with $\sigma(T)$ and $\sigma_{\omega}(T)$ connected, then there exists a compact operator $K$ with $\|K\|<\epsilon$ such that $T+K$ is strongly irreducible. They (see [7]) also proved that if $T$ is a Cowen-Douglas operator having unique (SI)-decomposition, then there exists a compact operator $K$ with $\|K\|<\epsilon$ such that $T+K$ is strongly irreducible. C.L. Jiang, S. Power, and Z.Y. Wang (see [11]) proved that if $T$ is a biquasitriangular operator with $\sigma(T)$ connected, then there exists a compact operator $K$ with $\|K\|<\epsilon$ such that $T+K$ is strongly irreducible.

The main result of this article is the theorem below.

Main Theorem. Let $T \in \mathcal{B}(\mathcal{H})$ be a quasitriangular operator with $\sigma(T)$ connected and let $\varepsilon>0$ be given. Then there exists a compact operator $K$ with $\|K\|<\epsilon$ such that $T+K$ is strongly irreducible.

\section{Preparation}

In order to prove the Main Theorem, we need to prepare some lemmas.

Lemma 2.1. Let $T \in \mathcal{B}(\mathcal{H})$ be an operator with $\operatorname{Ran} T$ nonclosed. Then there is an infinite dimensional subspace $\mathcal{H}_{0}$ (closed) of $\mathcal{H}$ such that $\mathcal{H}_{0} \cap \operatorname{Ran} T=\{0\}$.

Proof. We know that $\operatorname{Ran} T=\operatorname{Ran}\left(T T^{*}\right)^{1 / 2}$. Without loss of generality, assume that $T$ is positive and that $\operatorname{Ran} T$ is dense in $\mathcal{H}$. Let $E(*)$ be the spectral measure of $T$. It is easy to see that $E((0, t]) \neq 0$ for all $t>0$ and that $E((0,\|T\|])=$ I. Choose a sequence $\left\{t_{k}\right\}_{k \geq 0}$ of positive numbers decreasing to zero such that $t_{0}=\|T\|$ and such that $E\left(\left(t_{k}, t_{k-1}\right]\right) \neq 0$ for all $k \geq 1$. Write $E_{k}=E\left(\left(t_{k}, t_{k-1}\right]\right)$. Let $\mathcal{H}_{n}=\bigvee\left\{\operatorname{Ran} E_{(2 k-1) 2^{n-1}}: k \geq 1\right\}$. Then $\left\{\mathcal{H}_{n}\right\}_{n \geq 1}$ is a pairwise orthogonal family of subspaces and $\mathcal{H}=\bigoplus_{n \geq 1} \mathcal{H}_{n}$. Let $P_{n}$ be the projection onto $\mathcal{H}_{n}$, i.e. 
$P_{n}=\sum_{k \geq 1} E_{(2 k-1) 2^{n-1}}$. It follows that $P_{n} T=T P_{n}$. It is not difficult to show that $0 \in \sigma\left(\left.\bar{P}_{n} T\right|_{\mathcal{H}_{n}}\right)$. Hence we can take $x_{n} \in \mathcal{H}_{n} \backslash \operatorname{Ran}\left(\left.P_{n} T\right|_{\mathcal{H}_{n}}\right)$ for each $n \geq 1$. Let $\mathcal{H}_{0}=\bigvee\left\{x_{n}, n \geq 1\right\}$. Then $\operatorname{dim} \mathcal{H}_{0}=+\infty$ and $\mathcal{H}_{0} \cap \operatorname{Ran} T=\{0\}$. In fact, if $T y=x=\sum_{n \geq 1} \alpha_{n} x_{n}$, then $\alpha_{n} x_{n}=P_{n} x=P_{n} T y=P_{n} T P_{n} y$. So $\alpha_{n}=0$. And then $x=0$.

Remark. If there is an infinite dimensional linear submanifold $\mathcal{X}$ of $\mathcal{H}$ such that $\mathcal{X}$ $\cap \operatorname{Ran} T=\{0\}$, then it follows from Lemma 2.1 that there is an infinite dimensional subspace $\mathcal{H}_{0}$ of $\mathcal{H}$ such that $\mathcal{H}_{0} \cap \operatorname{Ran} T=\{0\}$.

Lemma 2.2. Let $A \in \mathcal{B}\left(\mathcal{H}_{1}, \mathcal{H}\right), B \in \mathcal{B}\left(\mathcal{H}_{2}, \mathcal{H}\right)$. Suppose that $\operatorname{Ran} B \subset \operatorname{Ran} A$ and suppose that $\mathcal{X}$ is an infinite dimensional linear submainfold of $\mathcal{H}$ such that $\mathcal{X} \cap \operatorname{Ran} A=\{0\}$. Given $\epsilon>0$, then there exists a compact operator $K \in \mathcal{K}\left(\mathcal{H}_{2}, \mathcal{H}\right)$ with $\|K\|<\epsilon$ such that $\operatorname{Ran} A \cap \operatorname{Ran}(B+K)=\{0\}$ and such that $\operatorname{Ker}(B+K)=\{0\}$.

Proof. By the remark above, find $\mathcal{H}_{0}<\mathcal{H}$ such that $\mathcal{H}_{0} \cap \operatorname{Ran} A=\{0\}$ and $\operatorname{dim} \mathcal{H}_{0}=\infty$. Take an injective $K \in \mathcal{K}\left(\mathcal{H}_{2}, \mathcal{H}\right)$ mapping $\mathcal{H}_{2}$ into $\mathcal{H}_{0}$ and with $\|K\|<\epsilon$. If $u=A x=(B+K) y=B y+K y$, it follows by $\operatorname{Ran} B \subset \operatorname{Ran} A$ that $K y \in \operatorname{Ran} A \cap \mathcal{H}_{0}=\{0\}$. So $K y=0$. Since $K$ is injective, $y=0$. Hence $\operatorname{Ran} A \cap \operatorname{Ran}(B+K)=\{0\}$. Similarly, $\operatorname{Ker}(B+K)=\{0\}$.

Lemma $2.3([7])$. Let $T \in \mathcal{B}(\mathcal{H})$. Suppose that $\operatorname{dim} \operatorname{Ker} T=1 \operatorname{dim} \operatorname{Ker} T=1$ and suppose that $\bigvee_{n \geq 1} \operatorname{Ker} T^{n}=\mathcal{H}$. Then $T$ is strongly irreducible.

Lemma 2.4. Set

$$
T=\left[\begin{array}{cc}
T_{1} & T_{12} \\
& T_{2}
\end{array}\right] \mathcal{H}_{1}
$$

where the entry omitted is 0 . Suppose that

(i) $\operatorname{dimKer} T_{1}=1, \bigvee_{n \geq 1} \operatorname{Ker} T_{1}^{n}=\mathcal{H}_{1}$,

(ii) $\bigvee \operatorname{Ker} T_{2}^{n}=\mathcal{H}_{2}$, $n \geq 1$

(iii) $\operatorname{Ker} T_{12} \cap \operatorname{Ker} T_{2}=\{0\}$,

(iv) $\operatorname{Ran} T_{1} \cap \operatorname{Ran}\left(T_{12} \mid \operatorname{Ker} T_{2}\right)=\{0\} .\left(\operatorname{Ran}\left(T_{12} \mid \operatorname{Ker} T_{2}\right)=T_{12}\left(\operatorname{Ker} T_{2}\right)\right.$.)

Then $T$ is strongly irreducible.

Proof. Suppose $T(x \oplus y)=0$, where $x \in \mathcal{H}_{1}, y \in \mathcal{H}_{2}$. Computation shows that $T_{2} y=0$ and $T_{1} x+T_{12} y=0$. By (iv), $T_{1} x=0$, i.e. $x \in \operatorname{Ker} T_{1}$, and $T_{12} y=0$. It follows from (iii) that $y=0$. So $\operatorname{Ker} T=\operatorname{Ker} T_{1}$. It is easy to show that $\bigvee_{n \geq 1} \operatorname{Ker} T^{n}=\mathcal{H}_{1}$. Suppose that $P$ is an idempotent commuting with $T$. Then $P T^{n}=T^{n} P$ for $n \geq 1$. So $P\left(\operatorname{Ker} T^{n}\right) \subset \operatorname{Ker} T^{n}$ for all $n \geq 1$. Thus $\mathcal{H}_{1} \in \operatorname{Lat} P$. Set

$$
P=\left[\begin{array}{cc}
P_{1} & P_{12} \\
& P_{2}
\end{array}\right] \mathcal{H}_{2}
$$

Then $P_{i}^{2}=P_{i}$ and $P_{i} T_{i}=T_{i} P_{i}, i=1,2$. By Lemma 2.3 and the condition (i), $P_{1}=$ $\left.I\right|_{\mathcal{H}_{1}}$ or 0 . Assume $P_{1}=0$ (otherwise, consider $I-P$ ). Computing the (1,2)-entry shows that $P_{12} T_{2}=T_{1} P_{12}+T_{12} P_{2}$. Let $y \in \operatorname{Ker} T_{2}$. It follows that $P_{2} y \in \operatorname{Ker} T_{2}$, and so $P_{2} T_{2}=T_{2} P_{2}$. So $T_{1} P_{12} y=-T_{12} P_{2} y \in \operatorname{Ran} T_{1} \cap \operatorname{Ran}\left(\left.T_{12}\right|_{\operatorname{Ker} T_{2}}\right)$. By the condition (iv), $P_{2} y=0$. Hence $P_{2}\left(\operatorname{Ker} T_{2}\right)=\{0\}$. If $x \in \operatorname{Ker} T_{2}^{2}$, then $T_{2} x \in \operatorname{Ker} T_{2}$. 
This shows that $T_{2} P_{2} x=P_{2} T_{2} x=0, P_{2} x \in \operatorname{Ker} T_{2}$. Thus $P_{2} x=P_{2}\left(P_{2} x\right)=0$. So $P_{2}\left(\operatorname{Ker} T_{2}^{2}\right)=\{0\}$. Inductively, $P_{2}\left(\operatorname{Ker} T_{2}^{n}\right)=\{0\}$ for all $n \geq 1$. By the condition (ii), $P_{2}=0$. So $P=P^{2}=0$, and $T$ is strongly irreducible.

Lemma 2.5. Suppose that $T \in \mathcal{B}(\mathcal{H})$ and that $T$ satisfies the following conditions:

(i) $0 \in \partial \sigma(T)$ (the boundary of $\sigma(T)$ ),

(ii) $\operatorname{Ker} T \subset \bigcap_{n \geq 1} \operatorname{Ran} T^{n}$,

(iii) $\bigvee_{n \geq 1} \operatorname{Ker} T^{n}=\mathcal{H}$.

Let $\epsilon>0$ be given. Then there exists a compact operator $K$ with $\|K\|<\epsilon$ such that $T+K$ is strongly irreducible.

Proof. By Lemma 2.3, we only need to show this lemma in the case that $\operatorname{dimKer} T>$ 1. Choose $x_{0} \in \operatorname{Ker} T \backslash\{0\}$. Let $\mathcal{N}_{\infty}=\operatorname{Ker} T \ominus \mathcal{C} x_{0}$. By the condition (ii), we can take $x_{k} \in \mathcal{N}_{\infty}^{\perp}$ such that $T x_{k}=x_{k-1}$ for each $k \geq 1$. Let $\mathcal{H}_{1}=\bigvee\left\{x_{k}: 1 \leq k<\right.$ $+\infty\}$. Then $\mathcal{H}_{1} \in \operatorname{Lat} T$ and $\mathcal{N}_{\infty} \subset \mathcal{H}_{1}^{\perp}$. Set

$$
T=\left[\begin{array}{cc}
T_{1} & T_{12} \\
& T_{2}
\end{array}\right] \mathcal{H}_{2}=\mathcal{H}_{1}^{\perp}
$$

It is easy to see that the following hold.

(1) $\operatorname{dimKer} T_{1}=1, \bigvee_{n \geq 1} \operatorname{Ker} T_{1}^{n}=\mathcal{H}_{1}$ and $\overline{\operatorname{Ran} T^{1}}=\mathcal{H}_{1}$

(2) $0 \in \partial \sigma\left(T_{1}\right)$ (this follows from $0 \in \partial \sigma(T)$ ).

(3) $\bigvee_{n \geq 1} \operatorname{Ker} T_{2}^{n}=\mathcal{H}_{2}$ (It follows from that $\bigcap_{n \geq 1} \overline{\operatorname{Ran} T_{2}^{* n}} \subset \bigcap_{n \geq 1} \overline{\operatorname{Ran} T^{* n}}=\{0\}$ ).

(4) $\mathcal{N}_{\infty} \subset \operatorname{Ker} T_{2}$ and $T_{12}\left(\mathcal{N}_{\infty}\right)=\{0\}$

(5) $\operatorname{Ker}\left(\left.T_{12}\right|_{\operatorname{Ker} T_{2} \ominus \mathcal{N}_{\infty}}\right)=\{0\}$ and $\operatorname{Ran} T_{1} \cap T_{12}\left(\operatorname{Ker} T_{2} \ominus \mathcal{N}_{\infty}\right)=\{0\}$.

Let $A$ be an operator mapping $\left(\mathcal{H}_{1} \ominus \operatorname{Ker} T_{1}\right) \oplus\left(\operatorname{Ker} T_{2} \ominus \mathcal{N}_{\infty}\right)$ into $\mathcal{H}_{1}$ such that $A(x \oplus y)=T_{1} x+T_{12} y$. By (5) above, $\operatorname{Ker} A=\{0\}$. Since $0 \in \partial \sigma\left(T_{1}\right)$ and $\overline{\operatorname{Ran} T^{1}}=\mathcal{H}_{1},\left.A\right|_{\mathcal{H}_{1} \ominus \operatorname{Ker} T_{1}}$ is unbounded from below. So $\operatorname{Ran} A$ is nonclosed. By Lemma 2.2, we can take a $B \in \mathcal{K}\left(\mathcal{N}_{\infty}, \mathcal{H}_{1}\right)$ with $\|B\|<\epsilon$ and $\operatorname{Ker} B=\{0\}$ such that $\operatorname{Ran} B \cap \operatorname{Ran} A=\{0\}$. Define

$$
K x= \begin{cases}B x, & x \in \mathcal{N}_{\infty}, \\ 0, & x \in \mathcal{N}_{\infty}^{\perp} .\end{cases}
$$

Then $K \in \mathcal{K}(\mathcal{H})$ and $\|K\|<\epsilon$. It is easy to see that

$$
T+K=\left[\begin{array}{cc}
T_{1} & C \\
& T_{2}
\end{array}\right] \mathcal{H}_{2}
$$

satisfies $\operatorname{Ker} C \cap \operatorname{Ker} T_{2}=\{0\}$ and $\operatorname{Ran} T_{1} \cap C\left(\operatorname{Ker} T_{2}\right)=\{0\}$. By Lemma 2.4, $T+K$ is strongly irreducible.

Lemma 2.6. Let $T$ be an operator acting on $\mathcal{H}$ satisfying the following conditions:

(i) $0 \in \partial \sigma(T)$ and $\bigvee \operatorname{Ker} T^{n}=\mathcal{H}$.

(ii) $\operatorname{Ker} T \cap\left(\bigcap_{n \geq 1} \operatorname{Ran}^{n \geq 1}\right)$ is closed and dimKerT $\ominus\left(\operatorname{Ker} T \cap\left(\bigcap_{n \geq 1} \operatorname{Ran} T\right)\right)<\infty$.

Let $\epsilon>0$ be given. Then there exists a $K \in \mathcal{K}(\mathcal{H})$ with $\|K\|<\epsilon$ such that $T+K$ is strongly irreducible.

Proof. Write $\mathcal{N}_{\infty}=\operatorname{Ker} T \cap\left(\bigcap_{n \geq 1} \operatorname{Ran} T^{n}\right)$. Denote $\operatorname{Ker} T \ominus \mathcal{N}_{\infty}=\mathcal{N}_{0}$. By the condition (ii), $\operatorname{dim} \mathcal{N}_{0}<+\infty$. For $k \geq 1$, we can inductively define $\mathcal{N}_{k}=\{x$ : 
$\left.T x \in \mathcal{N}_{k-1}, x \perp \mathcal{N}_{\infty}\right\}$. Since $\operatorname{dim} \mathcal{N}_{0}<+\infty, \mathcal{N}_{0} \cap \operatorname{Ran} T^{n_{0}}=\{0\}$ for some $n_{0}$. So $\mathcal{N}_{k}=\mathcal{N}_{0}$ when $n_{0} \leq k<+\infty$. Thus $\bigvee\left\{\mathcal{N}_{k}: k<+\infty\right\}=\mathcal{N}_{n_{0}}$. Denote it by $\mathcal{H}_{1}$. Then $\mathcal{H}_{1} \in$ Lat $T$ and $\operatorname{dim} \mathcal{H}_{1}(\stackrel{\text { def }}{=} m)<+\infty$. Set

$$
T=\left[\begin{array}{cc}
T_{1} & T_{12} \\
& T_{2}
\end{array}\right] \begin{gathered}
\mathcal{H}_{1} \\
\mathcal{H}_{2}=\mathcal{H}_{1}^{\perp}
\end{gathered}
$$

It is not difficult to show that

(1) $\operatorname{Ker} T_{2}=\mathcal{N}_{\infty} \subset \bigcap_{n \geq 1} \operatorname{Ran} T_{2}^{n}$.

(2) $\bigvee \operatorname{Ker} T_{2}^{n}=\mathcal{H}_{2}$.

$$
n \geq 1
$$

(3) $T_{1}^{m}=0$

Choose $C \in \mathcal{K}\left(\mathcal{H}_{1}\right)$ with $\|C\|<\frac{\epsilon}{2}\left(T_{1}+C\right)^{m-1} \neq 0$ and such that $\left(T_{1}+C\right)^{m}=0$. Take unit vectors $f \in \mathcal{H}_{1} \ominus \operatorname{Ran}\left(T_{1}+C\right)$ and $e \in \mathcal{N}_{\infty}$. Set

$$
K_{1}=\left[\begin{array}{cc}
C & \frac{\epsilon}{4} f \otimes e \\
0
\end{array}\right] \mathcal{H}_{1}
$$

where $(f \otimes e) x=(x, e) f$. Then $K_{1} \in \mathcal{K}(\mathcal{H})$ and $\left\|K_{1}\right\|<\frac{3 \epsilon}{4}$. It is not difficult to show that $\operatorname{Ker}\left(T+K_{1}\right)=\operatorname{Ker}\left(T_{1}+C\right) \oplus\left(\mathcal{N}_{\infty} \ominus \mathcal{C} e\right) \subset \bigcap \operatorname{Ran}\left(T+K_{1}\right)^{n}$. It is clear $n \geq 1$

that $0 \in \partial \sigma\left(T+K_{1}\right)$. By Lemma 2.5 , one can find a $K_{2} \in \mathcal{K}(\mathcal{H})$ with $\left\|K_{2}\right\|<\frac{\epsilon}{4}$ such that $T+K_{1}+K_{2}$ is strongly irreducible. Let $K=K_{1}+K_{2} \in \mathcal{K}(\mathcal{H})$. Then $\|K\|<\epsilon$.

Let $T \in \mathcal{K}(\mathcal{H})$ have the following form:

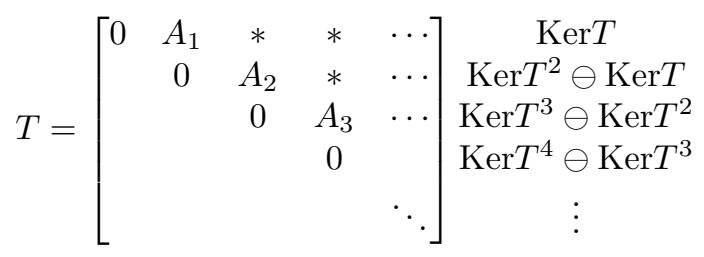

It is easy to see that $\operatorname{Ker} A_{i}=\{0\}$ and $\operatorname{Ker} T \cap \operatorname{Ran} T^{i}=\operatorname{Ran}\left(A_{1} A_{2} \cdots A_{i}\right)$ for all $i \geq 1$. Thus $\operatorname{Ker} T \cap\left(\bigcap_{n \geq 1} \operatorname{Ran} T^{n}\right)=\bigcap_{n \geq 1} \operatorname{Ran}\left(A_{1} A_{2} \cdots A_{i}\right)$. It follows that $\operatorname{Ker} T \cap\left(\bigcap_{n \geq 1} \operatorname{Ran} T^{n}\right)$ is closed when $\operatorname{Ran} A_{n}$ is closed for each $n \geq 1$.

Lemma 2.7. Let $T$ be as above. Suppose that $n_{0}$ is a natural number and suppose that $\mathcal{M}$ is an infinite dimensional subspace of $\operatorname{Ker} T^{n_{0}} \ominus \operatorname{Ker} T^{n_{0}-1}$ such that $\mathcal{M} \cap$ $\operatorname{Ran} A_{n_{0}}=\{0\}$. Let $\epsilon>0$ be given. Then there exists a compact operator $K$ with $\|K\|<\epsilon$ such that $T+K$ is strongly irreducible.

Proof. Without loss of generality, assume that $n_{0}>1$ and that $A_{i}$ has closed range with finite codimension for each $i<n_{0}$. Let $T_{1}=\left.T\right|_{\operatorname{Ker}^{n_{0}-1}}$, i.e.

$$
T_{1}=\left[\begin{array}{ccccc}
0 & A_{1} & \cdots & * & * \\
& 0 & \ddots & * & * \\
& & \ddots & & \vdots \\
& & & 0 & A_{n_{0}-2} \\
& & & & 0
\end{array}\right] \begin{gathered}
\operatorname{Ker} T \\
\operatorname{Ker} T^{2} \ominus \operatorname{Ker} T \\
\operatorname{Ker} T^{n_{0}-2} \ominus \operatorname{Ker} T^{n_{0}-3} \\
\operatorname{Ker} T^{n_{0}-2}
\end{gathered}
$$


Since Ran $A_{i}$ is closed for each $i<n_{0}, T_{1}$ is similar to $\bigoplus_{j=1}^{\infty} J_{j}$, where $J_{j}$ is a Jordon block for each $j$. So we can find $C_{1} \in \mathcal{K}\left(\operatorname{Ker} T^{n_{0}-1}\right)$ with $\left\|C_{1}\right\|<\frac{\epsilon}{2}$ such that $\operatorname{dim} \operatorname{Ker}\left(T_{1}+C_{1}\right)=1$ and $\bigvee_{n \geq 1} \operatorname{Ker}\left(T_{1}+C_{1}\right)^{n}=\operatorname{Ker} T^{n_{0}-1}$. It is clear that

$$
\operatorname{Ran}\left(T_{1}+C_{1}\right) \neq \overline{\operatorname{Ran}\left(T_{1}+C_{1}\right)}=\operatorname{Ker} T^{n_{0}-1} .
$$

Let $P$ be the projection onto $\left(\operatorname{Ker} T^{n_{0}-1}\right)^{\perp}$. Write $T_{2}=\left.P T\right|_{\operatorname{Ran} P}$. Then

$$
T_{2}=\left[\begin{array}{cccc}
0 & A_{n_{0}} & * & \cdots \\
& 0 & A_{n_{0}+1} & \cdots \\
& & 0 & \\
& & & \ddots
\end{array}\right] \begin{aligned}
& \mathcal{N}_{1}=\operatorname{Ker} T^{n_{0}} \ominus \operatorname{Ker} T^{n_{0}-1} \\
& \mathcal{N}_{2}=\operatorname{Ker} T^{n_{0}+1} \ominus \operatorname{Ker} T^{n_{0}} \\
& \mathcal{N}_{3}=\operatorname{Ker} T^{n_{0}+2} \ominus \operatorname{Ker} T^{n_{0}+1} \\
& \vdots
\end{aligned}
$$

Decompose $\mathcal{N}_{1}$ as $\mathcal{M} \oplus\left(\mathcal{N}_{1} \ominus \mathcal{M}\right)$. Then

$$
T_{2}=\left[\begin{array}{ccccc}
0 & 0 & B_{2} & * & \cdots \\
& 0 & B_{1} & * & \cdots \\
& & 0 & A_{n_{0}+1} & \cdots \\
& & 0 & \\
& & & & \ddots
\end{array}\right] \begin{aligned}
& \mathcal{M} \\
& \mathcal{N}_{1} \ominus \mathcal{M} \\
& \mathcal{N}_{2} \\
& \mathcal{N}_{3} \\
& \vdots
\end{aligned}
$$

where $\left[\begin{array}{l}B_{2} \\ B_{1}\end{array}\right]=A_{n_{0}}$. For each $0 \neq x \in \mathcal{N}_{2}, A_{n_{0}} x=B_{2} x+B_{1} x \notin \mathcal{M}$. So $B_{1} x \neq 0$. Set

$$
T_{3}=\left[\begin{array}{cccc}
0 & B_{1} & * & \cdots \\
& 0 & A_{n_{0}+1} & \cdots \\
& & 0 & \\
& & & \ddots
\end{array}\right] \begin{aligned}
& \mathcal{N}_{1} \ominus \mathcal{M} \\
& \mathcal{N}_{2} \\
& \mathcal{N}_{3} \\
& \vdots
\end{aligned}
$$

Then $\operatorname{Ker} T_{3}=\mathcal{N}_{1} \ominus \mathcal{M}$, and $T$ can be written as

$$
T=\left[\begin{array}{ccc}
T_{1} & T_{12} & * \\
& 0 & T_{23} \\
& & T_{3}
\end{array}\right] \begin{aligned}
& \mathcal{H}_{1}=\operatorname{Ker} T^{n_{0}-1} \\
& \mathcal{M} \\
& \mathcal{H}_{2}=\mathcal{H} \ominus\left(\mathcal{H}_{1} \oplus \mathcal{M}\right)
\end{aligned}
$$

where $\left.T_{23}\right|_{\operatorname{Ker} T_{3}}=0$. Take $C_{2} \in \mathcal{K}(\mathcal{M})$ with $\left\|C_{2}\right\|<\frac{\epsilon}{4}$ such that $\operatorname{dimKer} C_{2}=1$ and such that $\bigvee_{n \geq 1} \operatorname{Ker} C_{2}^{n}=\mathcal{M}$. By Lemma 2.2, choose $C_{3} \in \mathcal{K}\left(\mathcal{H}_{2}, \mathcal{M}\right)$ with $\left\|C_{3}\right\|<\frac{\epsilon}{8}$ such that $\operatorname{Ran} C_{3} \cap \operatorname{Ran} C_{2}=\{0\}$ and such that $\operatorname{Ker} C_{3}=\mathcal{H}_{2} \ominus \operatorname{Ker} T_{3}$. Take a rank one operator $C_{4} \in \mathcal{K}\left(\mathcal{M}, \mathcal{H}_{1}\right)$ with $\left\|C_{4}\right\|<\frac{\epsilon}{16}$ such that $\operatorname{Ker} C_{4}=\mathcal{M} \ominus \operatorname{Ker} C_{2}$, $\operatorname{Ker}\left(T_{12}+C_{4}\right) \cap \operatorname{Ker} C_{2}=\{0\}$ and $\left(T_{12}+C_{4}\right)\left(\operatorname{Ker} C_{2}\right) \cap \operatorname{Ran}\left(T_{1}+C_{1}\right)=\{0\}$. Set

$$
K=\left[\begin{array}{ccc}
C_{1} & C_{4} & 0 \\
& C_{2} & C_{3} \\
& & 0
\end{array}\right] \begin{gathered}
\mathcal{H}_{1} \\
\mathcal{M} \\
\mathcal{H}_{2}
\end{gathered}
$$

Then $K \in \mathcal{K}(\mathcal{H})$ and $\|K\|<\epsilon$, and so

$$
T+K=\left[\begin{array}{ccc}
T_{1+} C_{1} & T_{12}+C_{4} & * \\
& C_{2} & T_{23}+C_{3} \\
& & T_{3}
\end{array}\right] \begin{gathered}
\mathcal{H}_{1} \\
\mathcal{M} \\
\mathcal{H}_{2}
\end{gathered}
$$

Similarly to the proof of Lemma 2.4, one can with no difficulty verify that $T+K$ is strongly irreducible. 
Lemma 2.8. Suppose that $T$ has the form (3) and that the following conditions are satisfied:

(i) $\overline{\operatorname{Ran} A_{i}}=\operatorname{Ran} A_{i}$ and $\operatorname{dimKer} A_{i}^{*}<+\infty$ for each $i$.

(ii) $\operatorname{dimKer} T \cap \bigcap_{n>1}\left(\operatorname{Ran} T^{n}\right)=+\infty$.

(iii) $\operatorname{dimKer} T \ominus\left(\operatorname{Ker} T \cap\left(\bigcap_{n \geq 1} \operatorname{Ran} T^{n}\right)\right)=+\infty$.

Let $\epsilon>0$ be given. Then there exists a compact operator $K$ with $\|K\|<\epsilon$ such that $T+K$ is strongly irreducible.

Proof. Write $\mathcal{N}_{\infty}=\operatorname{Ker} T \cap\left(\bigcap_{n \geq 1} \operatorname{Ran} T^{n}\right)$ and $\mathcal{N}_{0}=\operatorname{Ker} T \ominus \mathcal{N}_{\infty}$. For $1 \leq k<+\infty$, inductively define $\mathcal{N}_{k}=\left\{x: T x \in \mathcal{N}_{k-1}, x \perp \mathcal{N}_{\infty}\right\}$, and set $\bigvee\left\{\mathcal{N}_{k}: 0 \leq k<\right.$ $+\infty\}=\mathcal{H}_{1}$. By (i) and (iii), there is an infinite dimensional linear submanifold $\mathcal{X}$ of $\mathcal{H}_{1}$ such that $\mathcal{X} \cap \operatorname{Ran} T=\{0\}$. It is clear that $\mathcal{H}_{1} \in \operatorname{Lat} T$. Set

$$
T=\left[\begin{array}{cc}
T_{1} & T_{12} \\
& T_{2}
\end{array}\right] \mathcal{H}_{1}^{\perp}
$$

It is easy to see that

(1) $\operatorname{Ker} T_{1}=\mathcal{N}_{0}$ and $\bigvee_{n \geq 1} \operatorname{Ker} T_{1}^{n}=\mathcal{H}_{1}$,

(2) $\mathcal{N}_{\infty} \subset \operatorname{Ker} T_{2}$ and $\bigvee_{n \geq 1} \operatorname{Ker} T_{2}^{n}=\mathcal{H}_{1}^{\perp}$.

Write $\mathcal{M}=A_{1}^{-1}\left(\mathcal{N}_{\infty}\right)$. Since $\operatorname{Ran} A_{1}$ is closed and $\operatorname{Ker} A_{1}=\{0\}$, we can find a positive number $r$ such that $r\|x\| \leq\left\|A_{1} x\right\|$ for $x \in \mathcal{M}$. Write $\mathcal{L}=P_{\mathcal{H}_{1}^{\perp}} \mathcal{M}$, where $P_{\mathcal{H}_{1}^{\perp}}$ is the projection onto $\mathcal{H}_{1}^{\perp}$. Suppose $x=x_{1} \oplus x_{2} \in \mathcal{M}, x_{1} \in \mathcal{H}_{1}, x_{2} \in \mathcal{L}$.

$$
\left\|P_{\mathcal{H}_{1}^{\perp}} x\right\|=\left\|x_{2}\right\| \geq \frac{\left\|T_{2} x_{2}\right\|}{\left\|T_{2}\right\|}=\frac{\left\|A_{1} x_{2}\right\|}{\left\|T_{2}\right\|} \geq \frac{r}{\left\|T_{2}\right\|}\|x\| .
$$

So $\mathcal{L}$ is closed. Moreover, $T_{12} y \in \operatorname{Ran} T_{1}$ for all $y \in \mathcal{L}$. Write $\mathcal{H}_{2}=\mathcal{H}_{1}^{\perp} \ominus \mathcal{N}_{\infty}$. Set

$$
T_{2}=\left[\begin{array}{cc}
0 & T_{23} \\
& T_{3}
\end{array}\right] \mathcal{N}_{\infty}
$$

It is easy to see that $\operatorname{Ker} T_{3}=\mathcal{L} \oplus\left(\operatorname{Ker} T_{2} \ominus \mathcal{N}_{\infty}\right)$ and that $\bigvee_{n \geq 1} \operatorname{Ker} T_{3}^{n}=\mathcal{H}_{2}$. If $0 \neq y \in \operatorname{Ker} T_{3} \ominus \mathcal{L}$, then $T_{12} y \notin \operatorname{Ran} T_{1}$. Notice that $\mathcal{X} \cap\left(\operatorname{Ran} T_{1}+T_{12}\left(\operatorname{Ker} T_{3} \ominus \mathcal{L}\right)\right) \subset$ $\mathcal{X} \cap \operatorname{Ran} T=\{0\}$ and that

$$
T=\left[\begin{array}{ccc}
T_{1} & 0 & \left.T_{12}\right|_{\mathcal{H}_{2}} \\
& 0 & T_{23} \\
& & T_{3}
\end{array}\right] \begin{aligned}
& \mathcal{H}_{1} \\
& \mathcal{N}_{\infty} \\
& \mathcal{H}_{2}
\end{aligned}=\left[\begin{array}{ccc}
0 & 0 & T_{23} \\
& T_{1} & T_{12} \mid \mathcal{H}_{2} \\
& & T_{3}
\end{array}\right] \begin{aligned}
& \mathcal{N}_{\infty} \\
& \mathcal{H}_{1} \\
& \mathcal{H}_{2}
\end{aligned}
$$

Similarly to the proof of Lemma 2.5 , by Lemma 2.2 there is a $C \in \mathcal{K}\left(\mathcal{H}_{2}, \mathcal{H}_{1}\right)$ with $\|C\|<\frac{\epsilon}{2}$ such that $\operatorname{Ker} C=\mathcal{H}_{2} \ominus \mathcal{L}$ and $\operatorname{Ran} C \cap\left(\operatorname{Ran} T_{1}+T_{12}\left(\operatorname{Ker} T_{3} \ominus \mathcal{L}\right)\right)=\{0\}$. Write $B=C+T_{12} \mid \mathcal{H}_{2}$. Then $\operatorname{Ran} T_{1} \cap B\left(\operatorname{Ker} T_{3}\right)=\{0\}$. Take $T_{0} \in \mathcal{K}\left(\mathcal{N}_{\infty}\right)$ with $\left\|T_{0}\right\|<\frac{\epsilon}{4}$ such that $\operatorname{dimKer} T_{0}=1$ and such that $\bigvee_{n \geq 1} \operatorname{Ker} T_{0}^{n}=\mathcal{N}_{\infty}$. Since $\operatorname{dim} \mathcal{N}_{\infty}=$ $+\infty, \operatorname{Ran} T_{0} \neq \overline{\operatorname{Ran} T_{0}}=\mathcal{N}_{\infty}$. By Lemma 2.2 , we can find a $D \in \mathcal{K}\left(\mathcal{H}_{1}, \mathcal{N}_{\infty}\right)$ with $\|D\|<\frac{\epsilon}{8}$ such that $\operatorname{Ran} D \cap \operatorname{Ran} T_{0}=\{0\}$ and $\operatorname{Ker} D=\mathcal{H}_{1} \ominus \operatorname{Ker} T_{1}$. Set

$$
K=\left[\begin{array}{ccc}
T_{0} & D & 0 \\
& 0 & C \\
& & 0
\end{array}\right] \begin{aligned}
& \mathcal{N}_{\infty} \\
& \mathcal{H}_{1} \\
& \mathcal{H}_{2}
\end{aligned}
$$


Then $K \in \mathcal{K}(\mathcal{H}),\|K\|<\epsilon$ and

$$
T+K=\left[\begin{array}{ccc}
T_{0} & D & * \\
& T_{1} & B \\
& & T_{3}
\end{array}\right] \begin{aligned}
& \mathcal{N}_{\infty} \\
& \mathcal{H}_{2}
\end{aligned}
$$

Similarly to the proof of Lemma 2.4 , one can show that $T+K$ is strongly irreducible.

By the equivalence of $(\text { str-v })_{-m}$ and (str-vi) ${ }_{-m}$ of Theorem 1.2 in [4], we have the following lemma.

Lemma 2.9 ([4]). Let $m$ be a natural number and let $T \in \mathcal{B}(\mathcal{H})$ be a quasitriangular operator with $\sigma(T)$ and $\sigma_{w}(T)$ connected. Let $\epsilon>0$ and $\lambda \in \sigma_{e}(T) \cup$ $\left(\bigcup_{k \geq m} \rho_{S-F}^{(k)}(T)\right)$ be given. Then there exist a $K \in \mathcal{K}(\mathcal{H})$ with $\|K\|<\epsilon$ and a sequence $\left\{P_{n}\right\}_{n \geq 0}$ of finite rank projections increasing to $I$ with respect to the strong operator topology with $\operatorname{rank} P_{n}=m n$ such that $\left(I-P_{n}\right)(T-\lambda+K) P_{n}=0$ for all $n \geq 0$, i.e.

$$
T+K-\lambda=\left[\begin{array}{lllll}
0 & * & * & * & \cdots \\
& 0 & * & * & \cdots \\
& & 0 & * & \cdots \\
& & & 0 & \\
& & & & \ddots
\end{array}\right] \begin{aligned}
& \operatorname{Ran} P_{1} \\
& \operatorname{Ran}\left(P_{2}-P_{1}\right) \\
& \operatorname{Ran}\left(P_{3}-P_{2}\right) \\
& \operatorname{Ran}\left(P 4-P_{3}\right) \\
& \vdots
\end{aligned}
$$

Theorem 2.1. Let $T \in \mathcal{B}(\mathcal{H})$ be a quasitriangular operator with $\sigma(T)$ and $\sigma_{w}(T)$ connected. Let $\epsilon>0$ be given. Then there exists a compact operator $K$ with $\|K\|<\epsilon$ such that $T+K$ is strongly irreducible.

Proof. Without loss of generality, assume that $0 \in \partial \sigma(T)$. By Lemma 2.9, find $K_{1} \in \mathcal{K}(\mathcal{H})$ with $\left\|K_{1}\right\|<\frac{\epsilon}{4}$ and a sequence $\left\{P_{n}^{(1)}\right\}_{n \geq 0}$ of finite rank projections increasing to $I$ with respect to the strong operator topology so that

$$
T+K_{1}=\left[\begin{array}{llll}
0 & * & * & \cdots \\
& 0 & * & \cdots \\
& & 0 & \\
& & & \ddots
\end{array}\right] \begin{aligned}
& \operatorname{Ran} P_{1}^{(1)} \\
& \operatorname{Ran}\left(P_{2}^{(1)}-P_{1}^{(1)}\right) \\
& \operatorname{Ran}\left(P_{3}^{(1)}-P_{2}^{(1)}\right) \\
& \vdots
\end{aligned}
$$

It is obvious that $\sigma_{p}\left(\left(T+K_{1}\right)^{*}\right) \subset\{0\}$. While $0 \in \sigma_{\text {lre }}(T)$,

$$
\sigma\left(T+K_{1}\right)=\sigma_{w}\left(T+K_{1}\right)=\sigma_{w}(T) .
$$

Write $P_{1}=P_{1}^{(1)}$ and $\mathcal{N}_{1}=\operatorname{Ran} P_{1}^{(1)}$. Let $T_{1}=\left.\left(I-P_{1}\right)\left(T+K_{1}\right)\right|_{\operatorname{Ran}\left(I-P_{1}\right)}$. It is not difficult to show that $\sigma\left(T_{1}\right)=\sigma_{w}\left(T_{1}\right)=\sigma_{w}(T), \sigma_{\text {lre }}\left(T_{1}\right)=\sigma_{\text {lre }}(T)$ and $\operatorname{ind}\left(T_{1}-\lambda\right)=\operatorname{ind}(T-\lambda)$ for all $\lambda \in \rho_{S-F}\left(T_{1}\right)$. Applying Lemma 2.9 to $T_{1}$, one can find a compact operator $K^{(2)} \in \mathcal{K}(\mathcal{H})$ with $\left\|K^{(2)}\right\|<\frac{\epsilon}{8}$ and a sequence $\left\{P_{n}^{(2)}\right\}_{n \geq 0}$ of finite rank projections increasing to $\left.I\right|_{\operatorname{Ran}\left(I-P_{1}\right)}$ with respect to the 
strong operator topology such that $\operatorname{rank} P_{1}^{(2)}=2 \operatorname{rank} P_{1}$ and

$$
T_{1}+K^{(2)}=\left[\begin{array}{llll}
0 & * & * & \cdots \\
& 0 & * & \cdots \\
& & 0 & \\
& & & \ddots
\end{array}\right] \begin{aligned}
& \operatorname{Ran} P_{1}^{(2)} \\
& \operatorname{Ran}\left(P_{2}^{(2)}-P_{1}^{(2)}\right) \\
& \vdots \\
& \vdots
\end{aligned}
$$

Since $\operatorname{rank} P_{2}^{(1)}<+\infty$, there is a natural number $n_{1}$ such that $\left\|\left(I-P_{2}\right) P_{2}^{(1)}\right\|<\frac{1}{2}$, where $P_{2}$ is the projection onto $\operatorname{Ran} P_{1} \oplus \operatorname{Ran} P_{n_{1}}^{(2)}$. Write $\mathcal{N}_{2}=\operatorname{Ran} P_{1}^{(2)}$ and $\mathcal{N}_{j}=\operatorname{Ran}\left(P_{j-1}^{(2)}-P_{j-2}^{(2)}\right)$ for $2<j \leq n_{1}+1$. Set

$$
K_{2}=\left[\begin{array}{ll}
0 & \\
& K^{(2)}
\end{array}\right] \begin{aligned}
& \operatorname{Ran} P_{1} \\
& \operatorname{Ran}\left(I-P_{1}\right)
\end{aligned}
$$

Then $K_{2} \in \mathcal{K}(\mathcal{H})$ and $\left\|K_{2}\right\|<\frac{\epsilon}{8}$. Let $T_{2}=\left.\left(I-P_{2}\right)\left(T+K_{1}+K_{2}\right)\right|_{\operatorname{Ran}\left(I-P_{2}\right)}$. One can show that $\sigma\left(T_{2}\right)=\sigma_{w}\left(T_{2}\right)=\sigma_{w}(T), \sigma_{l r e}\left(T_{2}\right)=\sigma_{\text {lre }}(T)$ and $\operatorname{ind}\left(T_{2}-\lambda\right)=\operatorname{ind}(T-\lambda)$ for all $\lambda \in \rho_{S-F}\left(T_{2}\right)$.

Repeatedly using the process above, we can inductively choose a sequence $\left\{n_{i}\right\}_{i \geq 1}$ of natural numbers, a sequence $\left\{\mathcal{N}_{k}\right\}_{k \geq 1}$ of pairwise orthogonal finite dimensional subspaces, an increasing sequence $\left\{P_{k}\right\}$ of finite rank projections and a sequence $\left\{K_{n}\right\}_{n \geq 1}$ of compact operators such that

(i) $\operatorname{dim} \mathcal{N}_{k} \leq \operatorname{dim} \mathcal{N}_{k+1}<+\infty(k \geq 1)$,

(ii) $\operatorname{Ran} P_{k}=\bigoplus\left\{\mathcal{N}_{j}: j \leq 1+\sum_{i=1}^{k-1} n_{i}\right\}(k \geq 1)$,

(iii) $\operatorname{dim} \mathcal{N}_{2+\sum_{i=1}^{k-1} n_{i}}=2^{k} \operatorname{rank} P_{k}(k \geq 1)$,

(iv) $\left\|\left(I-P_{n}\right) P_{n}^{(1)}\right\|<\frac{1}{n}$ for $n \geq 1$, and hence $\underset{1 \leq k<+\infty}{\bigoplus} \mathcal{N}_{k}=\mathcal{H}$.

(v) $\left\|K_{n}\right\|<\frac{\epsilon}{2^{n+1}}$, hence $\bar{K}_{1}=\sum_{1 \leq k<+\infty} K_{n} \in \mathcal{K}(\mathcal{H})$ and $\left\|\bar{K}_{1}\right\|<\frac{\epsilon}{2}$,

$$
T+\bar{K}_{1}=\left[\begin{array}{ccccc|c}
0 & B_{1} & * & * & \cdots & \mathcal{N}_{1} \\
& 0 & B_{2} & * & \cdots & \mathcal{N}_{2} \\
& & 0 & B_{3} & \cdots & \mathcal{N}_{3} \\
& & & 0 & \\
\mathcal{N}_{4} \\
\vdots
\end{array}\right.
$$

Since $\operatorname{dim} \mathcal{N}_{k} \leq \operatorname{dim} \mathcal{N}_{k+1}<+\infty$, we can choose $C_{k} \in \mathcal{K}\left(\mathcal{N}_{k+1}, \mathcal{N}_{k}\right)$ with $\left\|C_{k}\right\|<$ $\frac{\epsilon}{k+3}$ so that $B_{k}+C_{k}$ is surjective. Set

$$
C=\left[\begin{array}{cccc}
0 & C_{1} & & \\
& 0 & C_{2} & \\
& & 0 & \ddots \\
& & & \ddots
\end{array}\right] \begin{aligned}
& \mathcal{N}_{1} \\
& \mathcal{N}_{2} \\
& \mathcal{N}_{3} \\
& \vdots
\end{aligned}
$$

Then $C \in \mathcal{K}(\mathcal{H})$ and $\|C\|<\frac{\epsilon}{4}$. Write $\bar{K}_{2}=\bar{K}_{1}+C$. It is easy to see that

$$
\operatorname{dim}\left(\operatorname{Ker}\left(T+\bar{K}_{2}\right) \cap\left(\bigcap_{n \geq 1} \operatorname{Ran}\left(T+\bar{K}_{2}\right)^{n}\right)\right)=+\infty
$$


and that $\bigvee_{n \geq 1} \operatorname{Ker}\left(T+\bar{K}_{2}\right)^{n}=\mathcal{H}$. Set $T+\bar{K}_{2}$ in the form (3):

$$
T+\bar{K}_{2}=\left[\begin{array}{cccc}
0 & A_{1} & * & \cdots \\
& 0 & A_{2} & \\
& & 0 & \ddots \\
& & & \ddots
\end{array}\right] \begin{aligned}
& \operatorname{Ker}\left(T+\bar{K}_{2}\right) \\
& \operatorname{Ker}\left(T+\bar{K}_{2}\right)^{2} \ominus \operatorname{Ker}\left(T+\bar{K}_{2}\right) \\
& \operatorname{Ker}\left(T+\bar{K}_{2}\right)^{3} \ominus \operatorname{Ker}\left(T+\bar{K}_{2}\right)^{2} \\
& \vdots
\end{aligned}
$$

Consider each $A_{i}$. By Lemmas 2.6-2.8, we can find $\bar{K}_{3} \in \mathcal{K}(\mathcal{H})$ with $\left\|\bar{K}_{3}\right\|<\frac{\epsilon}{4}$ such that $T+\bar{K}_{2}+\bar{K}_{3}$ is strongly irreducible. Let $K=\bar{K}_{2}+\bar{K}_{3} \in \mathcal{K}(\mathcal{H})$. Then $\|K\|<\epsilon$. This completes the proof of Theorem 2.1.

Remark. In fact, Theorem 2.1 can be strengthened to the theorem below, and this will be useful in answering Question $\mathrm{H}$.

Theorem 2.1'. Let $T$ be a quasitriangular operator with $\sigma(T)$ and $\sigma_{w}(T)$ connected. Given $\epsilon>0$, then there exists a compact operator $K$ with $\|K\|<\epsilon$ such that

(i) $T+K$ is strongly irreducible,

(ii) $\sigma_{p}\left((T+K)^{*}\right)=\emptyset$,

(iii) $\operatorname{Ker} \tau_{B, T+K}=\{0\}$ if $\sigma_{p}(B)=\emptyset$, where $\tau_{B, T+K}$ is the Rosenblum operator.

Proof. Look back to the proof of Theorem 2.1. It is easy to see that $T+\bar{K}_{2}$ has dense range. We know that if $A=\left\{a_{i j}\right\}_{i j}$ is a triangular operator with respect to a suitable orthonormal basis, then $\sigma_{p}\left(A^{*}\right) \subset\left\{\bar{a}_{i i}: i\right\}$. Now we recall the proof of Lemmas 2.5-2.8.

(a) Look back to the formula (1) in the proof of Lemma 2.5. If RanT is dense in $\mathcal{H}$, then $\sigma_{p}\left(T_{1}^{*}\right)=\sigma_{p}\left(T_{2}^{*}\right)=\emptyset$. To see $(2)$, note that $\sigma_{p}\left((T+K)^{*}\right)=\emptyset$. Look back to the proof of Lemma 2.6. If $\operatorname{Ran} T$ is dense in $\mathcal{H}$, then $\operatorname{Ran}\left(T+K_{1}\right)$ is dense. Thus $\sigma_{p}\left((T+K)^{*}\right)=\emptyset$.

(b) Now look at (4), in the proof of Lemma 2.7. If $\overline{\operatorname{Ran} T}=\mathcal{H}$, then $\overline{\operatorname{RanT}}_{3}=$ $\mathcal{H}_{2}$ and $\sigma_{p}\left(T_{3}^{*}\right)=\emptyset$. In (5), it is clear that $\sigma_{p}\left(\left(T_{1}+C_{1}\right)^{*}\right)=\sigma_{p}\left(C_{2}^{*}\right)=\emptyset$. So $\sigma_{p}\left((T+K)^{*}\right)=\emptyset$.

(c) Look back to (6) in the proof of Lemma 2.8. Write $W=T_{12} P_{\mathcal{L}}$ and $V=$ $T_{12} P_{\mathcal{H}_{2} \ominus \mathcal{L}}$. Then $\left.T_{12}\right|_{\mathcal{H}_{2}}=W+V$. Look at (7). Set

$$
S=\left[\begin{array}{cc}
T_{1} & B \\
& T_{3}
\end{array}\right] \mathcal{H}_{1}
$$

If $\overline{\operatorname{Ran} T}=\mathcal{H}$, then $\overline{\operatorname{Ran} T_{3}}=\mathcal{H}_{2}$. So $\sigma_{p}\left(T_{3}^{*}\right)=\emptyset$. If $S^{*}(x \oplus y)=0$, where $x \in \mathcal{H}_{1}$ and $y \in \mathcal{H}_{2}$, then $T_{1}^{*} x=0$ and $B^{*} x+T_{3}^{*} y=0$. Notice that $B=C+W+V$. Since $\operatorname{Ran} W \subset \operatorname{Ran} T_{1}, W^{*} x=0$. Since $\operatorname{Ran} C^{*} \subset \mathcal{L}$ and $\operatorname{Ran} V^{*} \subset \mathcal{H}_{2} \ominus \mathcal{L}$, it follows that $C^{*} x=0$. Hence $V^{*} x+T_{3}^{*} y=0$, i.e. $T^{*}(x \oplus y)=0$. While $\overline{\operatorname{Ran} T}=\mathcal{H}, x \oplus y=0$. Thus $\sigma_{p}\left(S^{*}\right)=\emptyset$. It is clear that $\sigma_{p}\left(T_{0}^{*}\right)=\emptyset$. So $\sigma_{p}\left((T+K)^{*}\right)=\emptyset$.

Summarily, in the proof of Theorem 2.1, because $\operatorname{Ran}\left(T+\bar{K}_{2}\right)$ is dense in $\mathcal{H}$, not only is $T+K$ strongly irreducible, but also $\sigma_{p}\left((T+K)^{*}\right)=\emptyset$.

Now we are going to prove (iii). Without loss of generality, assume that $T+K(\stackrel{\text { def }}{=}$ A) can be written as

$$
A=\left[\begin{array}{ccc}
T_{1} & * & * \\
& T_{2} & * \\
& & T_{3}
\end{array}\right] \begin{gathered}
\mathcal{H}_{1} \\
\mathcal{H}_{3}
\end{gathered}
$$


where $\bigvee \operatorname{Ker}_{i}^{n}=\mathcal{H}_{i}, i=1,2,3$. Suppose $B X-X A=0$. Write $X=$ $n \geq 1$

$\left(X_{1}, X_{2}, X_{3}\right)$ where $X_{i}=\left.X\right|_{\mathcal{H}_{i}}$. Thus $B X_{1}-X_{1} T_{1}=0$ and hence $B^{n} X_{1}=X_{1} T_{1}^{n}$ for all $n \geq 1$. If $y \in \operatorname{Ker} T_{1}^{n}$, then $B^{n} X_{1} y=0$. By $\sigma_{p}(B)=\emptyset, X_{1} y=0$. So $X_{1}=0$. Similarly, $X_{2}=0$ and $X_{3}=0$, i.e. $X=0$.

By the upper semi-continuity of the spectrum, the continuity of index and Theorem 2.2 of [1] or Theorem 3.49 of [3], we have the following lemma.

Lemma 2.10 ([1], [3]). Suppose $\emptyset \neq \Gamma \subset \sigma_{l e}(T)$ and $\epsilon>0$. Then there exists a compact operator $K$ with $\|K\|<\epsilon$ such that

$$
T+K=\left[\begin{array}{rr}
N & * \\
& \tilde{T}
\end{array}\right] \quad \begin{aligned}
& \mathcal{H}_{2} \\
& \mathcal{H}_{2}
\end{aligned}
$$

where $N$ is a diagonal normal operator of uniform infinite multiplicity, $\sigma(N)=$ $\sigma_{\text {lre }}(N)=\Gamma, \sigma(\tilde{T})=\sigma(T), \sigma_{\text {lre }}(\tilde{T})=\sigma_{\text {lre }}(T)$, and $\operatorname{ind}(\tilde{T}-\lambda)=\operatorname{ind}(T-\lambda)$ for all $\lambda \in \rho_{S-F}(T)$.

Lemma 2.11 ([2], [3]). Let $A, B$ be two operators and let $\tau_{A, B}$ be the Rosenblum operator. Then the followings are equivalent:

(i) $\sigma_{r}(A) \cap \sigma_{l}(B)=\emptyset$.

(ii) $\tau_{A, B}$ is surjective.

(iii) $\operatorname{Ran} \tau_{A, B}$ contains all compact operators.

By Corollary 2.4 of [4], it is not difficult to prove the following lemma.

Lemma $2.12([4],[5])$. Suppose that $T \in \mathcal{B}(\mathcal{H})$ is quasitriangular and that $\sigma(T)=$ $\sigma_{w}(T)$. Let $\Gamma=\left\{\lambda_{n}\right\}_{n \geq 1} \subset \sigma(T)$ satisfying the following conditions:

(i) $\operatorname{Card}\left\{n: \lambda_{n}=\lambda_{j}\right\}=+\infty$ for all $j \geq 1$.

(ii) Each clopen of $\sigma(T)$ intersects with $\Gamma$.

Let $\epsilon>0$ e open. Then there exists a compact operator $K$ with $\|K\|<\epsilon$ such that $\bigvee\left\{\operatorname{Ker}\left(T+K-\lambda_{n}\right)^{k}: n \geq 1, k \geq 1\right\}=\mathcal{H}, \Gamma \subset \sigma_{p}(T+K)$ and $\sigma_{p}\left((T+K)^{*}\right)=\emptyset$.

Moreover, if $\sigma(T)$ and $\sigma_{w}(T)$ are connected, and if $\rho_{S-F}^{(n)}(T)$ contains a nonempty connected open subset $\Omega$, then $K$ can be chosen so that $T+K \in \mathcal{B}_{n}(\Omega)$.

Lemma $2.13([11])$. Let $T \in \mathcal{B}(\mathcal{H})$. Suppose $\sigma_{0}(T)=\emptyset$ and $\epsilon>0$. Then there exists a compact operator $K$ with $\|K\|<\epsilon$ such that

(i) $\sigma(T+K)=\sigma(T)$,

(ii) $\min \operatorname{ind}(T+K-\lambda)= \begin{cases}0, & \lambda \in \rho_{S-F}^{ \pm}(T), \\ 1, & \lambda \in \rho_{S-F}^{(0)}(T) \cap \sigma(T) \text {. }\end{cases}$

Lemma 2.14. Let $T \in \mathcal{B}(\mathcal{H})$. Suppose that $\sigma(T) \cap \rho_{S-F}(T)=\rho_{S-F}^{+}(T)$. Let $\left\{\Omega_{j}\right\}_{j}$ be the connected components of $\rho_{S-F}^{(1)}(T)$. Suppose that $\bigcup_{j} \Omega_{j}$ intersects with arbitary clopen of $\sigma(T)$. Let $\epsilon>0$ be given. Then there exists a $K \in \mathcal{K}(\mathcal{H})$ with $\|K\|<\epsilon$ such that

(i) $\bigvee\left\{\operatorname{Ker}(T+K-\lambda): \lambda \in \bigcup_{j} \Omega_{j}\right\}=\mathcal{H}$ and $\sigma_{p}\left((T+K)^{*}\right)=\emptyset$ 
(ii) $T+K$ has the form

$$
T+K=\left[\begin{array}{cccc}
B_{1} & & & \\
* * & B_{2} & & \\
* * & * & B_{3} & \\
\vdots & \vdots & & \ddots
\end{array}\right] \quad \begin{gathered}
\\
\\
\end{gathered}
$$

where each $B_{j}(j<\infty)$ is a Cowen-Douglas operator with index $1, \sigma\left(B_{i}\right) \cap \sigma\left(B_{j}\right)=$ $\emptyset(i \neq j, i, j<+\infty)$ and $\bigvee\left\{\mathcal{M}_{j}: k \leq j \leq+\infty\right\}$ is invariant under the commutant of $T+K(1 \leq k \leq+\infty)$.

Proof. Let $\sigma_{j}$ be the maximal connected closed subset of $\sigma(T)$ containing $\Omega_{j}$ for each $j$. Without loss of generality, assume that $\sigma_{i} \cap \sigma_{j}=\emptyset$ when $i \neq j$. Let $\Phi_{j}$ be the interior of the closure of $\Omega_{j}$. Take $\alpha_{j} \in \Omega_{j}$. Let $\mu_{j}$ be the probability measure supported by $\partial \Phi_{j}$ such that $\int \varphi(z) d \mu_{j}(z)=\varphi\left(\alpha_{j}\right)$ for those functions analytic in a neighbourhood of $\bar{\Phi}_{j}$. Let $M_{j}$ be the operator of multiplication by $z$ on $L^{2}\left(\mu_{j}\right)$. Let $H^{2}\left(\mu_{j}\right)$ be the span of all rational functions with poles outside $\bar{\Phi}_{j}$. Set

$$
M_{j}=\left[\begin{array}{cc}
M_{j}^{+} & * \\
& M_{j}^{-}
\end{array}\right] \begin{aligned}
& H^{2}\left(\mu_{j}\right) \\
& H^{2}\left(\mu_{j}\right)^{\perp}
\end{aligned}
$$

It is easy to show that

(1) $M_{j}$ is normal and $\sigma\left(M_{j}\right)=\sigma_{l r e}\left(M_{j}\right)=\partial \Phi_{j}$,

(2) $\sigma\left(M_{j}^{-}\right)=\bar{\Phi}_{j}$ and $M_{j}^{-} \in \mathcal{B}_{1}\left(\Phi_{j}\right)$.

Applying Lemma 2.10 to $T^{*}$, one can take $K_{1} \in \mathcal{K}(\mathcal{H})$ with $\left\|K_{1}\right\|<\frac{\epsilon}{2}$ such that

(3) $T+K_{1}=\left[\begin{array}{cc}T_{1} & * \\ & \bigoplus_{j}^{*} N_{j}\end{array}\right]$,

(4) $\sigma\left(T_{1}\right)=\sigma(T), \sigma_{\text {lre }}\left(T_{1}\right)=\sigma_{\text {lre }}(T)$ and $\operatorname{ind}(T-\lambda)=1$ for $\lambda \in \bigcup_{j} \Omega_{j}$.

(5) $N_{j}$ is diagonal normal and $\sigma\left(N_{j}\right)=\sigma_{l r e}\left(N_{j}\right)=\partial \Phi_{j}$ for each $j$.

Since $N_{j}, M_{j}$ are normal and $\sigma\left(N_{j}\right)=\sigma_{\text {lre }}\left(N_{j}\right)=\sigma_{\text {lre }}\left(M_{j}\right)=\sigma\left(M_{j}\right)$, there exists a compact operator $\bar{K}_{j}$ with $\left\|\bar{K}_{j}\right\|<\frac{\epsilon}{2^{j+3}}$ such that $N_{j}+\bar{K}_{j} \cong M_{j}$, where $\cong$ is the unitary equivalence relation. Thus there is a $K_{2} \in \mathcal{K}(\mathcal{H})$ with $\left\|K_{2}\right\|<\frac{\epsilon}{4}$ such that

$$
\begin{aligned}
& T+K_{1}+K_{2} \cong\left[\begin{array}{cc}
T_{1} & \\
& \bigoplus_{j}^{*} M_{j}
\end{array}\right] \\
& =\left[\begin{array}{ccc}
T_{1} & { }^{*} & * \\
& \bigoplus_{j} M_{j}^{+} & * \\
& & \bigoplus_{j} M_{j}^{-}
\end{array}\right] \\
& \stackrel{\text { def }}{=}\left[\begin{array}{ll}
T_{2} & T_{12} \\
& \bigoplus_{j} M_{j}^{-}
\end{array}\right]
\end{aligned}
$$

By Theorem 3.48 of [3], choose $K_{3} \in \mathcal{K}(\mathcal{H})$ with $\left\|K_{3}\right\|<\frac{\epsilon}{8}$ such that

$$
T+\sum_{j=1}^{3} K_{j} \cong\left[\begin{array}{ll}
T_{2}+C_{1} & * \\
& \bigoplus_{j}^{*} M_{j}^{-}
\end{array}\right]
$$


and $\sigma\left(T_{2}+C_{1}\right)=\sigma_{w}\left(T_{2}+C_{1}\right)=\sigma(T) \backslash \bigcup_{j} \Omega_{j}$. Notice that each clopen $\sigma$ of $\sigma\left(T_{2}+C_{1}\right)$ intersects with the closure of $\bigcup_{j} \Omega_{j}$. There is a subset $\left\{\lambda_{k}\right\}_{k \geq 1} \subset \bigcup_{j} \Omega_{j}$ such that

(6) $\sup _{k} \operatorname{dist}\left(\lambda_{k}, \sigma\left(T_{2}+C_{1}\right)\right)<\frac{\epsilon}{16}$ and $\lim _{k} \operatorname{dist}\left(\lambda_{k}, \sigma\left(T_{2}+C_{1}\right)\right)=0$.

(7) Each clopen of $\sigma\left(T_{2}+C_{1}\right)$ contains limit points of $\left\{\lambda_{k}\right\}_{k \geq 1}$.

Let $\Gamma=\left\{\mu_{k}\right\}_{k \geq 1}$ be a dense subset of all limit points of $\left\{\lambda_{k}\right\}_{k \geq 1}$. By Lemma 2.12, find $K_{4} \in \mathcal{K}(\mathcal{H})$ with $\left\|K_{4}\right\|<\frac{\epsilon}{16}$ such that

$$
T+\sum_{j=1}^{4} K_{j} \cong\left[\begin{array}{ll}
T_{2}+C_{1}+C_{2} & \stackrel{*}{ } \\
& \bigoplus_{j}^{*} M_{j}^{-}
\end{array}\right]
$$

where

$$
T_{2}+C_{1}+C_{2}=\left[\begin{array}{cccc}
v_{1} & * & * & \cdots \\
& v_{2} & * & \cdots \\
& & v_{3} & \\
& & & \ddots
\end{array}\right]
$$

with respect to a suitable orthonormal basis, where $v_{i} \in \Gamma$, and $\operatorname{Card}\left\{n: v_{n}=\mu_{j}\right\}$ $=+\infty$ for all $j \geq 1$. Choose $\lambda_{k_{j}}$ such that $\left|\lambda_{k_{j}}-v_{j}\right|<\frac{\epsilon}{2^{5} j}$ and $\lambda_{k_{j}} \notin\left\{\lambda_{k_{i}}\right\}_{i<j}$. Perturb $v_{j}$ by $\lambda_{k_{j}}-v_{j}$. Then one can find $K_{5} \in \mathcal{K}(\mathcal{H})$ with $\left\|K_{5}\right\|<\frac{\epsilon}{32}$ such that

$$
T+\sum_{j=1}^{5} K_{j} \cong\left[\begin{array}{cc}
T_{2}+\sum_{i=1}^{3} C_{i} & * \\
& \bigoplus_{j} M_{j}^{-}
\end{array}\right]
$$

where $\sigma_{0}\left(T_{2}+\sum_{i=1}^{3} C_{i}\right)=\left\{\lambda_{k_{j}}\right\}_{j \geq 1}, \underset{j}{\bigvee} \operatorname{Ker}\left(T_{2}+\sum_{i=1}^{3} C_{i}-\lambda_{k_{j}}\right)$ is equal to the acting space of $T_{2}+\sum_{i=1}^{3} C_{i}$, and $\operatorname{rank} E\left(\lambda_{k_{j}}, T_{2}+\sum_{i=1}^{3} C_{i}\right)=1$. Write $\bar{T}=T_{2}+\sum_{i=1}^{3} C_{i}$. Without loss of generality, assume that $\lambda_{k_{j}}=\lambda_{j}$ and that

$$
T+\sum_{j=1}^{5} K_{j}=\left[\begin{array}{ll}
\bar{T} & { }^{*} \\
& \bigoplus_{j} M_{j}^{-}
\end{array}\right] \begin{gathered}
\mathcal{M}^{\perp} \\
\mathcal{M}
\end{gathered}
$$

Notice that $\sigma_{p}\left(\left(T+\sum_{i=1}^{5} K_{i}\right)^{*}\right) \subset\left\{\bar{\lambda}_{j}: j \geq 1\right\}$. If $\sigma_{p}\left(\left(T+\sum_{i=1}^{5} K_{i}\right)^{*}\right)$ is nonempty, denote it by $\left\{\bar{a}_{j}: j\right\}$. Write $\mathcal{H}_{1}=\bigvee\left\{\operatorname{Ker}\left(\bar{T}-a_{j}\right): j\right\}, \mathcal{H}_{2}=\mathcal{M}^{\perp} \ominus \mathcal{H}_{1}$. Then $T+\sum_{j=1}^{5} K_{j}$ can be written as

$$
T+\sum_{j=1}^{5} K_{j}=\left[\begin{array}{ccc}
A_{1} & A_{12} & A_{13} \\
& A_{2} & A_{23} \\
& & \bigoplus_{j} M_{j}^{-}
\end{array}\right] \begin{gathered}
\mathcal{H}_{1} \\
\mathcal{H}_{2} \\
\mathcal{M}
\end{gathered}
$$

Notice that $\bigcup_{k} \operatorname{Ran}\left(A_{1}-a_{k}\right) \neq \mathcal{H}_{1}$ and that $\bigcup_{k} \operatorname{Ran}\left(\bigoplus_{j} M_{j}^{-}-a_{k}\right)^{*} \neq \mathcal{M}$. Choose unit vectors $e \in \mathcal{H}_{1} \backslash \bigcup_{k} \operatorname{Ran}\left(A_{1}-a_{k}\right)$ and $f \in \mathcal{M} \backslash \bigcup_{k} \operatorname{Ran}\left(\bigoplus_{j} M_{j}^{-}-a_{k}\right)^{*}$. Define 
$K_{6} x=\frac{\epsilon}{64}(x, f) e$, where $(x, f)$ is the scalar product of $x$ and $f$. Let $K=\sum_{j=1}^{6} K_{j} \epsilon$ $\mathcal{K}(\mathcal{H})$. Then $\|K\|<\epsilon$. It is an exercise to show that $\sigma_{p}\left((T+K)^{*}\right)=\emptyset$ and that $\bigvee\left\{\operatorname{Ker}(T+K-\lambda): \lambda \in \bigcup_{j} \Omega_{j}\right\}=\mathcal{H}$. Let $\mathcal{N}_{j}=\bigvee\left\{\operatorname{Ker}(T+K-\lambda): \lambda \in \bigcup_{i \geq j} \Omega_{j}\right\}$ and $\mathcal{M}_{\infty}=\bigcap_{j<\infty} \mathcal{N}_{j}$. Write $\mathcal{M}_{j}=\mathcal{N}_{j} \ominus \mathcal{N}_{j+1}$ for $1 \leq j<+\infty$. Then

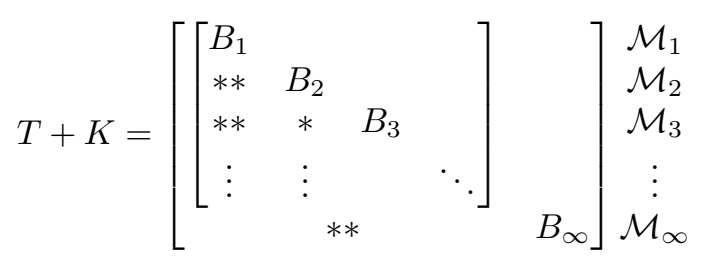

It is clear that $B_{j} \in \mathcal{B}_{1}\left(\Omega_{j}\right)$ for $j<\infty$ and that $\sigma\left(B_{j}\right) \subset \sigma_{j}$. Furthermore, if $X$ commutes with $T+K$, then $X$ has the form

$$
X=\left[\begin{array}{cccc}
X_{1} & & & \\
* * & X_{2} & & \\
* * & * & X_{3} & \\
\vdots & \vdots & & \ddots
\end{array}\right] \begin{aligned}
& \\
& \\
&
\end{aligned}
$$

\section{Proof of Main Theorem}

By Theorem 2.1 and Lemma 2.13, assume that $\sigma_{w}(T)$ is nonconnected and

$$
\min \operatorname{ind}(T-\lambda)= \begin{cases}0, & \lambda \in \rho_{S-F}^{+}(T) \\ 1, & \lambda \in \sigma(T) \cap \rho_{S-F}^{(0)}(T) .\end{cases}
$$

Suppose $\left\{\Omega_{j}\right\}_{j}$ are the connected components of $\sigma(T) \cap \rho_{S-F}^{(0)}(T)$. Let $\mathcal{H}_{l}=$ $\bigvee\left\{\operatorname{Ker}(T-\lambda)^{*}: \lambda \in \bigcup_{j} \Omega_{j}\right\}$. Then $T$ can be written as

$$
T=\left[\begin{array}{cc}
\overline{T_{1}} & * \\
& \overline{T_{2}}
\end{array}\right] \begin{gathered}
\mathcal{H}_{l}^{\perp} \\
\mathcal{H}_{l}
\end{gathered}
$$

By Lemma 2.10, choose a $\bar{K}_{1} \in \mathcal{K}\left(\mathcal{H}_{l}\right)$ with $\left\|\bar{K}_{1}\right\|<\frac{\epsilon}{4}$ such that

$$
\bar{T}_{2}+\bar{K}_{1}=\left[\begin{array}{cc}
N & * \\
& \overline{T_{3}}
\end{array}\right] \begin{aligned}
& \mathcal{H}_{0} \\
& \mathcal{H}_{l} \ominus \mathcal{H}_{0}
\end{aligned}
$$

where $N$ is a diagonal normal operator of uniform infinite multiplicity, $\sigma(N)=$ $\sigma_{\text {lre }}(N)=\sigma_{\text {lre }}\left(\bar{T}_{2}\right), \sigma\left(\bar{T}_{3}\right)=\sigma\left(\bar{T}_{2}\right), \sigma_{\text {lre }}\left(\bar{T}_{3}\right)=\sigma_{\text {lre }}\left(\bar{T}_{2}\right)$ and $\operatorname{ind}\left(\bar{T}_{3}-\lambda\right)=$ $\operatorname{ind}\left(\bar{T}_{2}-\lambda\right)=-1$ for $\lambda \in \bigcup_{j} \Omega_{j}$. Write $\mathcal{H}_{1}=\mathcal{H}_{l}^{\perp} \oplus \mathcal{H}_{0}$. Set

$$
K_{1}=\left[\begin{array}{ll}
0 & \\
& \bar{K}_{1}
\end{array}\right] \begin{aligned}
& \mathcal{H}_{l}^{\perp} \\
& \mathcal{H}_{l}
\end{aligned}
$$


Then $K_{1}$ is compact and $\left\|K_{1}\right\|<\frac{\epsilon}{4}$. Look at $T+K_{1}$ :

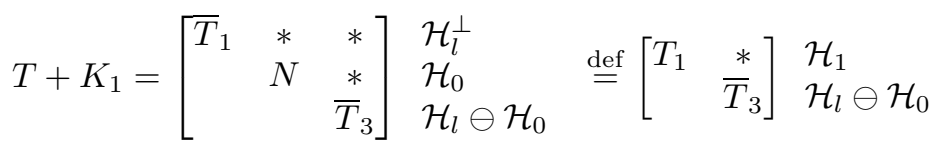

It is clear that $\sigma\left(T_{1}\right)=\sigma_{w}\left(T_{1}\right)=\sigma(T)$, ind $\left(T_{1}-\lambda\right)>0$ for $\lambda \in \rho_{S-F}\left(T_{1}\right) \cap \sigma\left(T_{1}\right)$ and $\operatorname{ind}\left(T_{1}-\lambda\right)=1$ for $\lambda \in \bigcup_{j} \Omega_{j}$. By Lemma 2.12 , take $\bar{K}_{2} \in \mathcal{K}\left(\mathcal{H}_{1}\right)$ with $\left\|\bar{K}_{2}\right\|<\frac{\epsilon}{8}$ such that $A=T_{1}+\bar{K}_{2} \in \mathcal{B}_{1}\left(\Omega_{1}\right)$. Hence $A$ is strongly irreducible. Notice that $\mathcal{H}_{l}=\bigvee\left\{\operatorname{Ker}(T-\lambda)^{*}: \lambda \in \bigcup_{j} \Omega_{j}\right\}$. Each clopen of $\sigma\left(\bar{T}_{2}\right)$ intersects with some $\Omega_{j}$. So each clopen of $\sigma\left(\bar{T}_{3}\right)$ contains some $\Omega_{j}$. Notice that $\sigma\left(\bar{T}_{3}^{*}\right) \cap \rho_{S-F}\left(\bar{T}_{3}^{*}\right)=\rho_{S-F}^{+}\left(\bar{T}_{3}^{*}\right)$ and ind $\left(\bar{T}_{3}-\lambda\right)^{*}=1$ for $\lambda \in \bigcup_{j} \Omega_{j}$. Applying Lemma 2.14 to $\bar{T}_{3}^{*}$, find a compact operator $\bar{K}_{3}$ with $\left\|\bar{K}_{3}\right\|<\frac{\epsilon}{16}$ such that $\sigma_{p}\left(\bar{T}_{3}+\bar{K}_{3}\right)=\emptyset$ and $\bar{T}_{3}+\bar{K}_{3}$ can be written as

$$
\bar{T}_{3}+\bar{K}_{3}=\left[\left[\begin{array}{cccc}
B_{1} & * & * & \cdots \\
& B_{2} & * & \cdots \\
& & B_{3} & \\
& & & \ddots
\end{array}\right] \quad * \begin{array}{c} 
\\
\\
\end{array}\right.
$$

where each $B_{j}^{*}(j<+\infty)$ is a Cowen-Douglas operator with index $1, \sigma\left(B_{i}\right) \cap$ $\sigma\left(B_{j}\right)=\emptyset$ when $i \neq j, i, j<+\infty$, and $\bigoplus_{i=1}^{k} \mathcal{M}_{j}$ is invariant under the commutant of $\bar{T}_{3}+\bar{K}_{3}$ for each $1 \leq k \leq+\infty$. Set

$$
K_{2}=\left[\begin{array}{ll}
\bar{K}_{2} & \\
& \bar{K}_{3}
\end{array}\right] \begin{aligned}
& \mathcal{H}_{1} \\
& \mathcal{H}_{1} \ominus \mathcal{H}_{0}
\end{aligned} \in \mathcal{K}(\mathcal{H})
$$

Then $\left\|K_{2}\right\|<\frac{\epsilon}{8}$ and

$$
\begin{aligned}
& T+K_{1}+K_{2}=\left[\begin{array}{cc}
A & { }^{*} \\
& \bar{T}_{3}+\bar{K}_{3}
\end{array}\right] \begin{array}{l}
\mathcal{H}_{1} \\
\mathcal{H}_{1} \ominus \mathcal{H}_{0}
\end{array}
\end{aligned}
$$

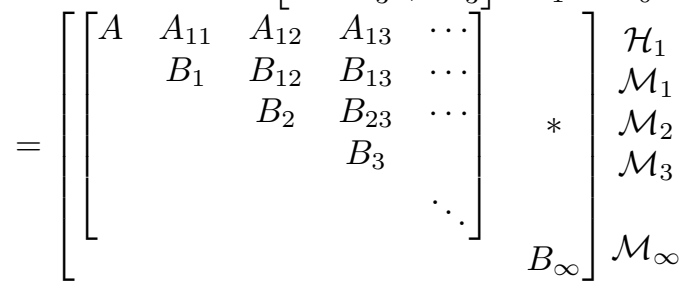

Because $\sigma\left(B_{i}\right) \cap \sigma\left(B_{j}\right)=\emptyset$ for $i \neq j$ and $\sigma_{\text {lre }}(A) \cap \sigma_{\text {lre }}\left(B_{j}\right)=\sigma_{\text {lre }}\left(B_{j}\right) \neq \emptyset$ for all $j<+\infty$, by Lemma 2.11, we can inductively find $C_{j} \in \mathcal{K}\left(\mathcal{M}_{j}, \mathcal{H}_{1}\right)$ with $\left\|C_{j}\right\|<\epsilon / 2^{j+4}$ such that $A_{11}+C_{1} \notin \operatorname{Ran} \tau_{A, B_{1}}$ and

$$
\left[\begin{array}{c}
A_{1, j+1}+C_{j+1} \\
B_{1, j+1} \\
\vdots \\
B_{j, j+1}
\end{array}\right] \notin \operatorname{Ran} \tau_{A_{j}, B_{j+1}}
$$


where

$$
A_{j}=\left[\begin{array}{cccc}
A & A_{11}+C_{1} & \cdots & A_{1, j}+C_{j} \\
& B_{1} & \cdots & B_{1, j} \\
& & \ddots & \vdots \\
& & & B_{j}
\end{array}\right] \mathcal{H}_{l}
$$

Write $D_{j}=A_{1, j}+C_{j}$. Define $K_{3} x=\sum_{j<+\infty} C_{j} P_{\mathcal{M}_{j}} x$, where $P_{\mathcal{M}_{j}}$ is the projection onto $\mathcal{M}_{j}$ for each $j<+\infty$. Then $K=K_{1}+K_{2}+K_{3}$ is compact and $\|K\|<\epsilon$. Moreover,

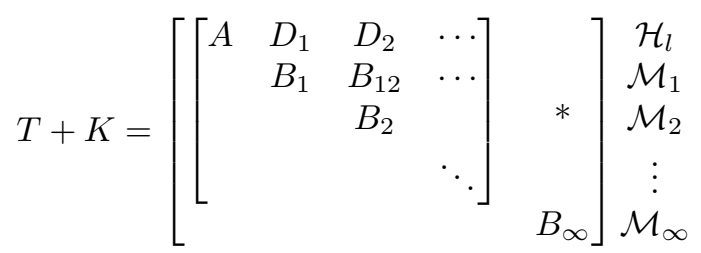

Now we are going to prove that $T+K$ is strongly irreducible. Suppose that $P$ is an idempotent operator commuting with $T+K$. Set

$$
P=\left[\begin{array}{cc}
P_{0} & Q_{01} \\
Q_{10} & \bar{P}
\end{array}\right] \begin{gathered}
\mathcal{H}_{1} \\
\mathcal{H}_{1}^{\perp}
\end{gathered}
$$

It is easy to see that $\left(\bar{T}_{3}+\bar{K}_{3}\right) Q_{10}=Q_{10} A$. So $\left(\bar{T}_{3}+\bar{K}_{3}-\lambda\right) Q_{10}=Q_{10}(A-\lambda)$ for $\lambda \in \Omega_{1}$. Since $A \in \mathcal{B}_{1}\left(\Omega_{1}\right)$ and $\sigma_{p}\left(\bar{T}_{3}+\bar{K}_{3}\right)=\emptyset, Q_{10}=0$. Furthermore, $\bar{P}$ is an idempotent operator commuting with $\bar{T}_{3}+\bar{K}_{3}$. So $\bar{P}$ has the form

$$
\bar{P}=\left[\begin{array}{cccc}
P_{1} & P_{12} & P_{13} & \cdots \\
& P_{2} & P_{23} & \cdots \\
& & P_{3} & \\
& & & \ddots
\end{array}\right] \quad * \begin{gathered}
\\
\\
\end{gathered}
$$

It is clear that $P_{i}$ is an idempotent operator commuting with $B_{i}$ for each $1 \leq i<$ $+\infty$, and that $P_{0}$ is idempotent and commutes with $A$. Since all $B_{j}(j<+\infty)$ and $A$ are strongly irreducible, $P_{i}$ is equal to either zero or the unit operator on its acting space for each $0 \leq j<+\infty$. Set

$$
P=\left[\left[\begin{array}{cccc}
P_{0} & P_{01} & P_{02} & \cdots \\
& P_{1} & P_{12} & \cdots \\
& & P_{2} & \\
& & & \ddots
\end{array}\right] * \begin{array}{c} 
\\
\\
\end{array}\right.
$$

If $P_{0}=0$, then $A P_{01}+D_{1} P_{1}=P_{01} B_{1}$. Since $D_{1} \notin \operatorname{Ran} \tau_{A, B_{1}}, P_{1}=0$. It follows from $P^{2}=P$ that $P_{01}=0$. Similarly, one can inductively prove that $P_{j}=0$ for $j<+\infty$ and that $P_{i, j}=0$ for $i, j<\infty$. Thus

$$
\bar{P}=\left[\begin{array}{cc}
0 & * \\
& P_{\infty}
\end{array}\right] \begin{aligned}
& \mathcal{H}_{1}^{\perp} \ominus \mathcal{M}_{\infty} \\
& \mathcal{M}_{\infty}
\end{aligned}
$$


It is clear that $\operatorname{Ran} \bar{P}^{*} \cap \operatorname{Ker}\left(\bar{T}_{3}+\bar{K}_{3}-\lambda\right)^{*}=\{0\}$ for all $\lambda \in \bigcup_{j} \Omega_{j}$. So $\bar{P}=0$. Hence $P=0$. If $P_{0}$ is the unit operator acting on $\mathcal{H}_{1}$, then one can show that $P=I$. So $T+K$ is strongly irreducible.

\section{REFERENCES}

[1] C. Apostol, C. Foias and D. Voiculescu, Some results on nonquasitriangular operators II, Rev. Roumaine Math. Pures et Appl. 18 (1973), 159-181. MR 48:12109a

[2] L. A. Fialkow, $A$ note on the range of the operator $X \rightarrow A X-X B$, Illinois J. Math. 25 (1981), 112-124. MR 84b:47021

[3] D. A. Herrero, Approximation of Hilbert space operators, I, 2nd ed., Pitman Research Notes in Math., 224. Longman Group UK Limited, 1989. MR 91k:47002

[4] - The diagonal entries in the formula 'quasitriangular-compact = triangular', and restrictions of quasitriangularity, Trans. Amer. Math. Soc. 298 (1986), 1-42. MR 88c:47022

[5] Spectral pictures of operators in the Cowen-Douglas class $\mathcal{B}_{n}(\Omega)$ and its closure, J. Operator Theory 18 (1987), 213-222. MR 89b:47032

[6] D. A. Herrero, C. L. Jiang, Limits of strongly irreducible operators and the Riesz decomposition theorem, Michigan. Math. J. 37 (1990), 283-291. MR 91k:47035

[7] Y. Q. Ji, C. L. Jiang and Z. Y. Wang, The strongly irreducible operators in the nest algebras, Integral Equations and Operator Theory 28 (1997), 28-44. MR 98b:47057

[8] Essentially normal + small compact $=$ strongly irreducible, Chinese Math. Ann. Series B 18 (1997), 485-494. MR 98i:47011

[9] The $(\mathcal{U}+\mathcal{K})$-orbit of essentially normal operators and compact perturbation of strongly irreducible operators, Functional Analysis in China, Math. and Its Applications, Vol. 356, Kluwer, Dordrecht, 1996, pp. 307-314. MR 97h:47013

[10] C. L. Jiang, S. H. Sun and Z. Y. Wang, Essentially normal operator + compact operator = strongly irreducible operator, Trans. Amer. Math. Soc. 349 (1997), 217-233. MR 97h:47012

[11] C. L. Jiang, S. Power and Z. Y. Wang, Biquasitriangular + small compact = strongly irreducible, J. London Math. Soc. (to appear)

Department of Mathematics, Jilin University, Changchun 130023, P.R. China 